\title{
Socially Responsible Design in the Context of International Development
}

\author{
A. Dearden, L. Dunckley, M. Best, S. Dray, A. Light, and J. Thomas
}

\section{Introduction}

Human beings evolved for many millennia; during most of that time, our major social contacts were within small, tightly knit groups who shared a common language, culture and physical context. Now, we find ourselves to be a part of a global community. Though we still have very different cultures, languages, perspectives, and physical contexts, we also share a planet with limited and shrinking resources and we share many interactions in our intellectual, technological and economic spheres. Potentially, technology offers many benefits to the many peoples of the world. Yet, there is also potential for damaging the diversity in the ecology of ideas and cultures that may be indispensable for humankind to survive the next millennium. More immediately, technology developed without sufficient understanding and involvement of those to be most affected by it will probably fail at best and in the worst cases, not only fail to provide anticipated benefits but produce negative side-effects. One such side-effect may well be making future technological usage more difficult.

While User-Centered Design and Participatory Design are important techniques within a cultural context in order to provide solutions that are useful, usable and acceptable, these approaches are particularly vital when there is a large discrepancy between the background knowledge and assumptions of end users and designers. In such cases, not only is it useful, it is, indeed, necessary to gain a deeper and broader understanding of people, users, contexts, and tasks. However, this understanding will always be filtered, and can all-to-easily be distorted and obscured by the unconscious cultural lenses of people from so-called "developed" countries when they are the developers of technology. Further complicating the problem, methods such as ethnography and participative design may have to be significantly modified or even replaced by entirely new and locally invented methods more appropriate to the specifics of situation, locale and/or culture.

There are great challenges involved in attempting to provide a socially responsible design process in the context of international development. However, these situations also provide potential benefits beyond those of development in less challenging circumstances. Since these circumstances are so novel, they also provide a potential breeding ground for new insights and methods of general applicability to HCI.

Most previous innovations were developed within the confines of one cultural context (although often spread to other cultures). Largely unexplored is the territory of creativity possible when the perspectives, strengths, and knowledge of multiple disparate cultures are brought to bear simultaneously. It is entirely possible to use ICT to strengthen and maintain local cultures rather than to accelerate a global lack of diversity. The panel authors listed above are organizing and running a workshop on this topic at CHI 2007 where over fifty participants from around the globe, including 
participants from both so-called "developed" and "developing" countries, will be sharing their experiences and lessons learned in the area of user-centered design and international development ("UCD4D"). Based on our experiences at this workshop, we will make a final determination on the composition of the panel presenters at INTERACT in order to provide the broadest possible range of perspectives.

\section{Participants}

Dr. Michael Best, a computer scientist by training with graduate degrees from the MIT Media Lab, he is an Assistant Professor at the Sam Nunn School of International Affairs at Georgia Tech University. Dr. Susan Dray, an experienced consultant in HCI with extensive experience in the area of international development. Professor Lynne Dunckley, Director of Research at the Institute for Information Technology at TVU in the UK. Dr. John Thomas, Research Staff Member at IBM's T. J. Watson Research Center has been interested in cross-cultural issues in HCI since organizing workshops in this area in 1992 and 1993.

We also plan to include four international participants based on a CHI workshop on this topic; ideally, one each from South America, Africa, Asia and Indonesia. Panel participants will share the specifics of their experiences of combining user centered design for international development as well as various perspectives on guidelines, methodologies and general patterns that may be applicable for international development. We will also discuss what future steps and linkages we feel are necessary for UCD4D to influence processes more deeply. Ultimately, our goal is to involve interested INTERACT participants in this process.

\section{Position Statements}

Michael Best asks: Does the Internet empower communities in central Ghana or the Mekong Delta of Vietnam? I look at the broad issues of whether the Internet is useful for economic or social development or whether it exacerbates political divisions.

Susan Dray will discuss experiences doing international user research in a number of so-called "developing" countries, sharing insights about how to capitalize on crosscultural research teams, as well as how to modify research methods to better "fit" across cultures.

Lynne Dunckley will discuss her experiences in researching usability methods in different cultural contexts and her current involvement in the development of ICT applications in sub-Saharan Africa.

John Thomas (main contact: jcthomas@us.ibm.com) will serve as moderator and provide a perspective on the interaction of cultures based on patterns of learning from The Walking People, a transcribed oral history of a branch of the Iroquois. In the multi-millennia long migration as recounted in this work by Paula Underwood, the group continually meets and interacts with cultures vastly different from their own. During all this time, they not only deal with immediate survival but constantly strive to learn general patterns of learning. 